\title{
Transport solide dans un cours d'eau en climat semi-aride : cas du bassin versant de l'Oued Boumessaoud (nord-ouest de l'Algérie)
}

\section{Sediment transport in a semi-arid stream: case of the wadi Boumessaoud (NWAlgeria)}

\author{
Sid Ahmed Bouguerra, Abderrazak Bouanani et Kamila Baba-Hamed
}

Volume 29, numéro 3, 2016

Reçu le 21 octobre 2014, accepté le 8 juin 2016

URI : https://id.erudit.org/iderudit/1038923ar

DOI : https://doi.org/10.7202/1038923ar

Aller au sommaire du numéro

Éditeur(s)

Université du Québec - INRS-Eau, Terre et Environnement (INRS-ETE)

ISSN

1718-8598 (numérique)

Découvrir la revue

Citer cet article

Bouguerra, S. A., Bouanani, A. \& Baba-Hamed, K. (2016). Transport solide dans un cours d'eau en climat semi-aride : cas du bassin versant de l'Oued

Boumessaoud (nord-ouest de l'Algérie). Revue des sciences de l'eau / Journal of Water Science, 29(3), 179-195. https://doi.org/10.7202/1038923ar

\section{Résumé de l'article}

L'exploitation des valeurs instantanées de débits liquides et de débits solides en suspension dans le bassin versant de l'oued Boumessaoud $\left(118 \mathrm{~km}^{2}\right)$, pour la période allant de septembre 1988 à août 2004, a permis de mettre en évidence la variabilité annuelle et saisonnière de l'apport solide, et d'examiner

l'ampleur du phénomène pendant les périodes d'érosion active de l'année dans ce bassin des régions méditerranéennes semi-arides où la contribution des crues s'est élevée à $71 \%$ durant la saison de l'automne, pour atteindre $97 \%$ de la charge globale moyenne au printemps. Les résultats de cette analyse montrent que la dégradation spécifique moyenne est de l'ordre de 518 $\mathrm{t} \cdot \mathrm{km}^{-2} \cdot \mathrm{an}^{-1}$. L'approche statistique abordée, qui est une régression reliant les deux variables débit liquide $\left(Q_{L}\right)$ et débit solide $\left(Q_{S}\right)$, a été testée à différentes échelles temporelles; les modèles générés sont de type puissance $\left(Q_{S}=a Q_{L}{ }^{b}\right)$, où les débits liquides expliquent globalement plus de $80 \%$ de la variance du transport solide. L'identification des périodes productives de sédiments dans cette dynamique érosive a été étudiée en analysant l'évolution de la concentration des particules en suspension dans le cours d'eau. Cette modélisation aura pour application la reconstitution de données manquantes au droit des stations de contrôle au niveau du bassin d'étude.
Tous droits réservés @ Revue des sciences de l'eau, 2016

Ce document est protégé par la loi sur le droit d'auteur. L’utilisation des services d'Érudit (y compris la reproduction) est assujettie à sa politique d'utilisation que vous pouvez consulter en ligne.

https://apropos.erudit.org/fr/usagers/politique-dutilisation/ 


\section{TRANSPORT SOLIDE DANS UN COURS D'EAU EN CLIMAT SEMI-ARIDE : CAS DU BASSIN VERSANT DE L'OUED BOUMESSAOUD (NORD-OUEST DE L'ALGÉRIE)}

Sediment transport in a semi-arid stream: case of the wadi Boumessaoud (NWAlgeria)

SID AHMED BOUGUERRA*, ABDERRAZAK BOUANANI, KAMILA BABA-HAMED

Université de Tlemcen, Faculté de Technologie, Département d'Hydraulique, BP 230, 13000 Tlemcen, Algérie

Reçu le 21 octobre 2014, accepté le 8 juin 2016

\section{RÉSUMÉ}

L'exploitation des valeurs instantanées de débits liquides et de débits solides en suspension dans le bassin versant de l'oued Boumessaoud $\left(118 \mathrm{~km}^{2}\right)$, pour la période allant de septembre 1988 à août 2004, a permis de mettre en évidence la variabilité annuelle et saisonnière de l'apport solide, et d'examiner l'ampleur du phénomène pendant les périodes d'érosion active de l'année dans ce bassin des régions méditerranéennes semiarides où la contribution des crues s'est élevée à $71 \%$ durant la saison de l'automne, pour atteindre $97 \%$ de la charge globale moyenne au printemps. Les résultats de cette analyse montrent que la dégradation spécifique moyenne est de l'ordre de $518 \mathrm{t} \cdot \mathrm{km}^{-2} \cdot \mathrm{an}^{-1}$. L'approche statistique abordée, qui est une régression reliant les deux variables débit liquide $\left(Q_{L}\right)$ et débit solide $\left(Q_{S}\right)$, a été testée à différentes échelles temporelles; les modèles générés sont de type puissance $\left(Q_{S}=a Q_{L}^{b}\right)$, où les débits liquides expliquent globalement plus de $80 \%$ de la variance du transport solide. L'identification des périodes productives de sédiments dans cette dynamique érosive a été étudiée en analysant l'évolution de la concentration des particules en suspension dans le cours d'eau. Cette modélisation aura pour application la reconstitution de données manquantes au droit des stations de contrôle au niveau du bassin d'étude.

Mots-clés : Transport solide, modélisation, débit liquide, débit solide, hystérésis.

\begin{abstract}
The analysis of instantaneous water discharge and suspended sediment loads in the watershed of the Boumessaoud Wadi $\left(118 \mathrm{~km}^{2}\right)$, for the period from September 1988 to August 2004, revealed the existence of annual and seasonal variability in sediment transport in this Mediterranean semiarid catchment and demonstrated the impact of flood events, which contribute to about $71 \%$ of the overall solid tonnage in the autumn and up to $97 \%$ in the spring. The results of this analysis indicate that the annual average specific erosion is about $518 \mathrm{t} \cdot \mathrm{km}^{-2} \cdot \mathrm{y}^{-1}$. The methodological approach adopted consists of finding a regression model capable of explaining the sediment load $\left(Q_{S}\right)$ as a function of the discharge $\left(Q_{L}\right)$,
\end{abstract}


tested by studying this relation at various temporal scales. The produced models are a power relationship type $\left(Q_{S}=a Q_{I}^{b}\right)$, where water discharge explains overall more than $80 \%$ of the variance in sediment yield. The identification of periods that are sediment-productive in this dynamic erosion system was studied by analysis of the evolution of the sediment concentrations in the stream. The objective of this modeling is to overcome the shortcomings of the sediment transport data available for our studied catchment.

Key Words: Sediment transport, modeling, liquid flows, sediment load, hysteresis.

\section{INTRODUCTION}

Le transport des matières solides en suspension est un phénomène qui a suscité d'énormes efforts de la part des nations, en matière de lutte contre la dégradation des terres arables, l'envasement des barrages et l'effet dévastateur des cycles d'inondation et de désertification, qu'il est capable de générer (DE PLOEY et al., 1991; SHABAN et KHAWLIE, 1998). La préservation de l'environnement a longtemps incité la communauté scientifique à faire valoir les résultats des recherches dans ce domaine par une mise en valeur des projets et programmes de gestion et de contrôle de l'érosion hydrique. En Algérie, lors des événements extrêmes, l'ordre de grandeur des concentrations moyennes varie de 50 à $150 \mathrm{~g} \cdot \mathrm{L}^{-1}$ et peut atteindre parfois $600 \mathrm{~g} \cdot \mathrm{L}^{-1}$ (MEDJBER, 2011).

Les effets du ruissellement sont intensifiés lorsque le sol est dénudé (labours, surpâturage, pratiques agricoles inadéquates, teneur en matière organique réduite des agrégats, incendie de forêt) (BOU KHEIR et al., 2001). Dans le bassin de l'oued Boumessaoud, à l'instar des zones semi-arides du nord de l'Algérie, l'érosion hydrique pose de nombreux problèmes liés à l'évaluation de la dégradation spécifique et du transport solide. Cette problématique a motivé beaucoup de chercheurs à comprendre les mécanismes de la morphogenèse des versants et l'hydrodynamisme des oueds pour tenter de quantifier les volumes des sédiments ainsi transportés. Plusieurs auteurs ont publié des études sur le phénomène (ACHITE et MEDDI, 2004; BOUANANI, 2004; BOUROUBA, 1998; ELAHCENE et al., 2012; GHENIM, 2008; MEGNOUNIF, 2007; PROBST et AMIOTTE SUCHET, 1992; SOGREAH, 1967; TERFOUS et al., 2001). Une de leurs priorités a été la recherche de modèles statistiques mettant en évidence la relation entre les paramètres hydroclimatiques et géomorphologiques et le transport des matériaux solides. Ces modèles sont applicables à des régions ou à des bassins versants où les mesures sont rares ou inexistantes (ACHITE, 2002; ELAHCENE et REMINI, 2009). Notre travail entre dans le cadre de cette thématique et comporte deux parties : la première consiste à évaluer le taux de dégradation spécifique au sein du bassin d'étude, la deuxième portera sur une analyse statistique à différentes échelles temporelles des couples de valeurs $\left(Q_{I}, Q_{S}\right)$ de la série de mesures disponibles en vue de rechercher la meilleure modélisation.

\section{MATÉRIEL ET MÉTHODE}

\subsection{Présentation $d u$ bassin versant}

Le bassin versant de l'oued Boumessaoud fait partie du grand bassin de la Tafna qui s'étend au nord-ouest de l'Algérie. Situé entre les longitudes $1^{\circ} 20^{\prime}$ et $1^{\circ} 30^{\prime} \mathrm{O}$, et les latitudes $34^{\circ} 51^{\prime} 15^{\prime \prime}$ et $35^{\circ} 05^{\prime} \mathrm{N}$ (Figure 1), il occupe une superficie de $118 \mathrm{~km}^{2}$ avec un périmètre de $59 \mathrm{~km}$, de forme assez allongée $\left(K_{G}=1,52\right)$ et d'un indice de pente global $I_{g}=0,036$ lui offrant un relief assez marqué selon la classification de l'ORSTOM (DUBREUIL et GUISCAFRE, 1971). Dans la partie sud du bassin, les pentes sont fortes ( $>20 \%$ ) et les altitudes atteignent 1150 m au niveau de Djebel Tefatisset et du plateau de Terny. Par contre, en direction du nord-ouest, depuis la plaine d'Henaya jusqu'au plateau de Zénata au droit de son exutoire, le bassin versant change de morphologie là où les pentes deviennent douces $(<5 \%)$ et les altitudes diminuent pour atteindre $150 \mathrm{~m}$ comme valeur minimale. La courbe hypsométrique indique que $80 \%$ de la surface se situe entre 900 et $200 \mathrm{~m}$ avec une altitude médiane égale à $630 \mathrm{~m}$. L'oued Boumessaoud long de $23,6 \mathrm{~km}$, dont la pente moyenne du cours d'eau principal est de $3,5 \%$, forme un réseau hydrographique assez dense avec ses multiples ramifications. Les valeurs de la densité de drainage ainsi que la fréquence des cours d'eau (représentée par le rapport du nombre total de cours d'eau à la surface du bassin) sont respectivement $2,39 \mathrm{~km}^{-1}$ et $3,68 \mathrm{~km}^{-2}$.

Du point de vue géologique, le bassin versant dans sa partie sud est occupé par les formations carbonatées jurassiques des monts de Tlemcen représentées par les dolomies de Tlemcen et de Terny, qui surmontent les bancs de grés de Boumediene au niveau de la forêt de Zarifet. Au centre, on trouve des formations marneuses et gréseuses du Tertiaire, des alluvions récentes du Pliocène et une carapace calcaire qui s'étale sur une grande partie du plateau de Zénata. Le nord du bassin est caractérisé par une dépression où affleurent des marnes du miocène inférieur et des alluvions récentes. Le climat qui règne dans cette région est de type semi-aride, la température moyenne est de $16,3{ }^{\circ} \mathrm{C}$. Le régime pluviométrique est très irrégulier et le bassin reçoit annuellement entre 226 et $630 \mathrm{~mm}$ avec une moyenne interannuelle de $396 \mathrm{~mm}$ à la station d'Henaya (1972-2010) (Figure 2). Deux périodes climatiques caractérisent l'année hydrologique : une période correspondant 


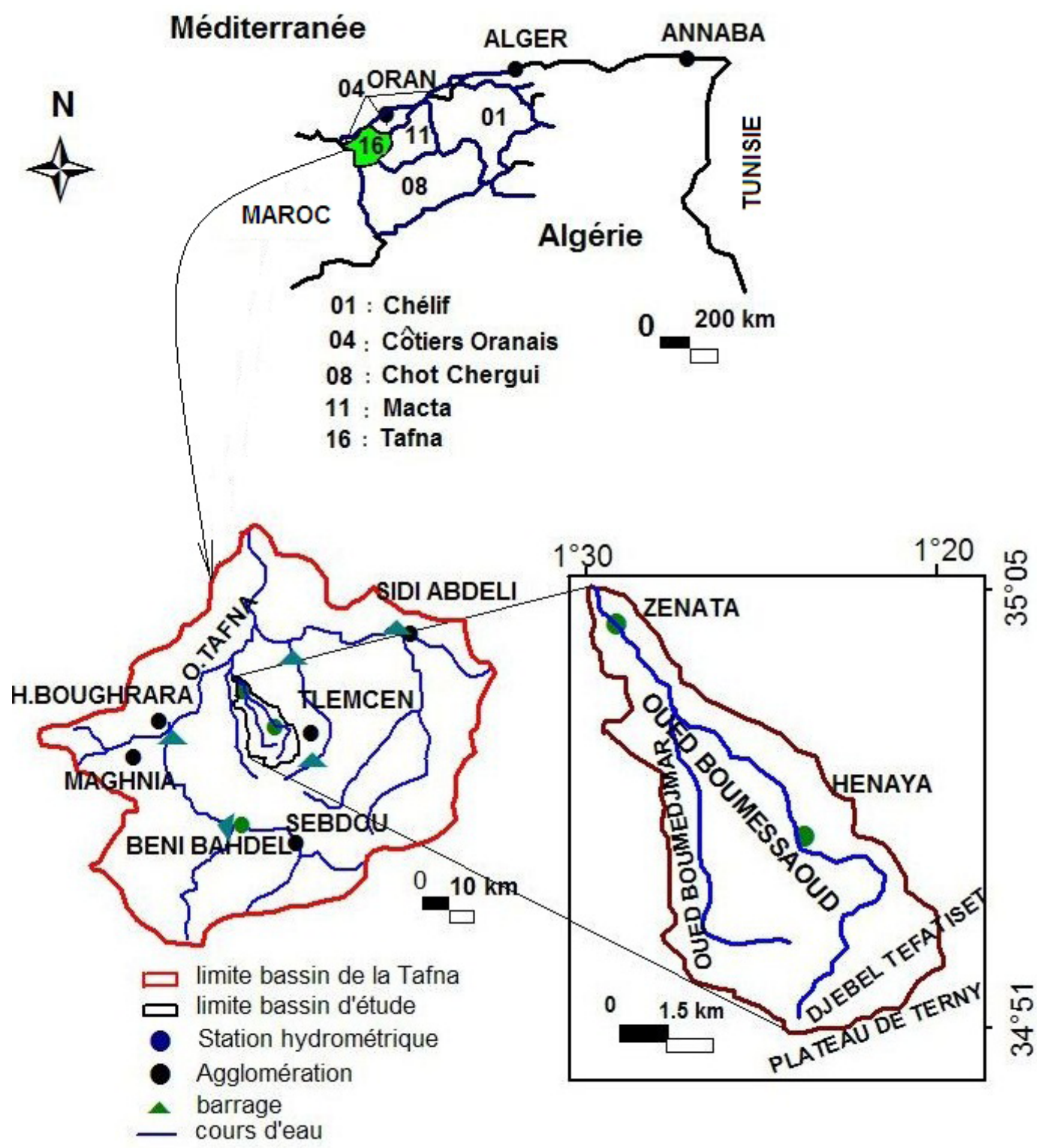

Figure 1. Situation géographique du bassin versant de l'oued Boumessaoud (nord-ouest de l'Algérie). Geographical location of the watershed of wadi Boumessaoud (NW Algeria).

aux mois secs de l'année (juin, juillet, août) et une autre pour les neuf mois restants dans laquelle les mois de novembre à mars sont considérés comme les plus pluvieux de l'année (Figure 3). Le débit moyen annuel spécifique écoulé durant la période allant de 1988/89 à 2004/05 à la station hydrométrique d'Henaya est estimé à $0,0058 \mathrm{~m}^{3} \cdot \mathrm{s}^{-1} \cdot \mathrm{km}^{-2}$.

\subsection{Données et méthodologies}

La station hydrométrique d'Henaya (latitude $=34^{\circ} 54^{\prime} 25^{\prime \prime} \mathrm{N}$, longitude $=1^{\circ} 23^{\prime} 58^{\prime \prime} \mathrm{O}, Z=552 \mathrm{~m}$ ) située sur l'axe de l'oued principal du bassin à environ $10 \mathrm{~km}$ de l'exutoire, contrôle une surface de $46 \mathrm{~km}^{2}$. Cette station de l'ANRH (Agence Nationale des Ressources Hydrauliques) couvrant une période allant de 1988 à 2004 permet de fournir les données nécessaires à l'étude, soit les mesures instantanée des débits liquides $Q_{L}\left(\mathrm{~m}^{3} \cdot \mathrm{s}^{-1}\right)$ et des débits solides $Q_{S}\left(\mathrm{~kg} \cdot \mathrm{s}^{-1}\right)$. L'autre station disponible dans le bassin versant est celle de Zénata située à l'exutoire et fournit uniquement des données de débits journaliers.

Notons que les débits liquides sont obtenus à partir de la courbe de tarage et que les débits solides sont le produit du débit liquide par la concentration des sédiments en suspension $\left(C_{S}\right)$ correspondante. Ces concentrations sont le résultat d'une procédure communément adaptée à toutes les stations hydrométriques appartenant à l'ANRH : à chaque hauteur d'eau lue sur une échelle limnimétrique, on prélève un échantillon d'eau chargée à la surface libre de l'écoulement du côté de la rive de l'oued au moyen d'un flacon de $500 \mathrm{ml}$. On transfère l'échantillon au laboratoire pour filtrage, séchage (à $100{ }^{\circ} \mathrm{C}$ en moyenne) et enfin pesée des sédiments ainsi 


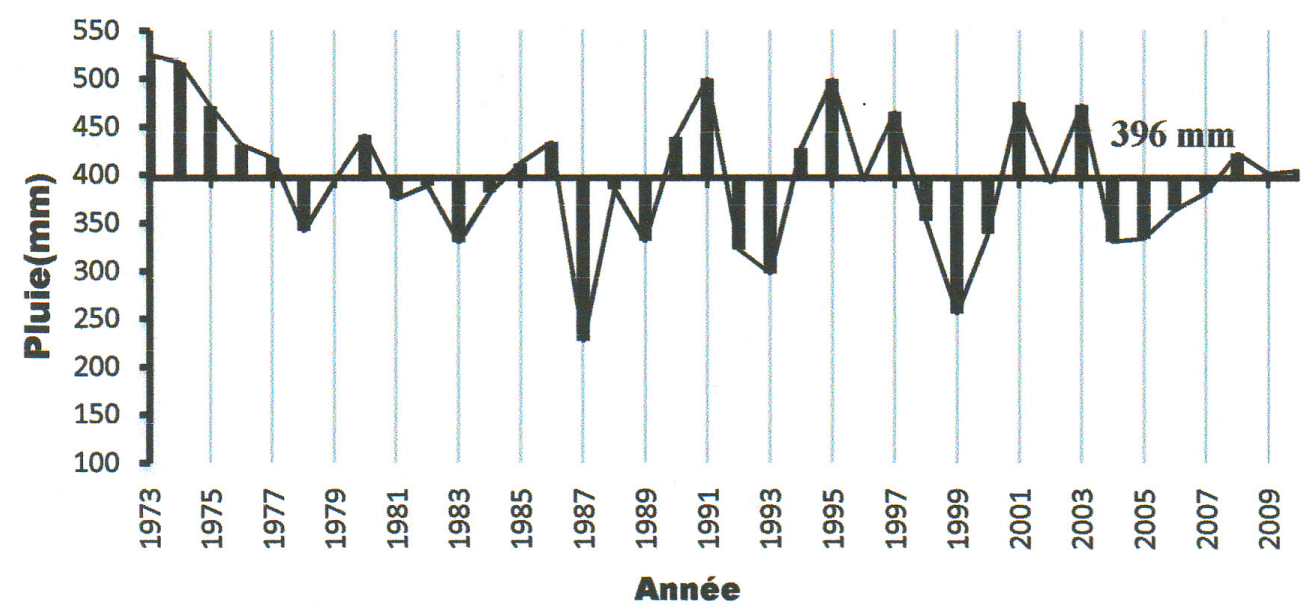

Figure 2. Variabilité interannuelle de la pluviométrie dans le bassin de l'oued Boumessaoud durant la période 1973-2010.

Interannual variability of the pluviometry in the watershed of wadi Boumessaoud during the period 1973-2010.

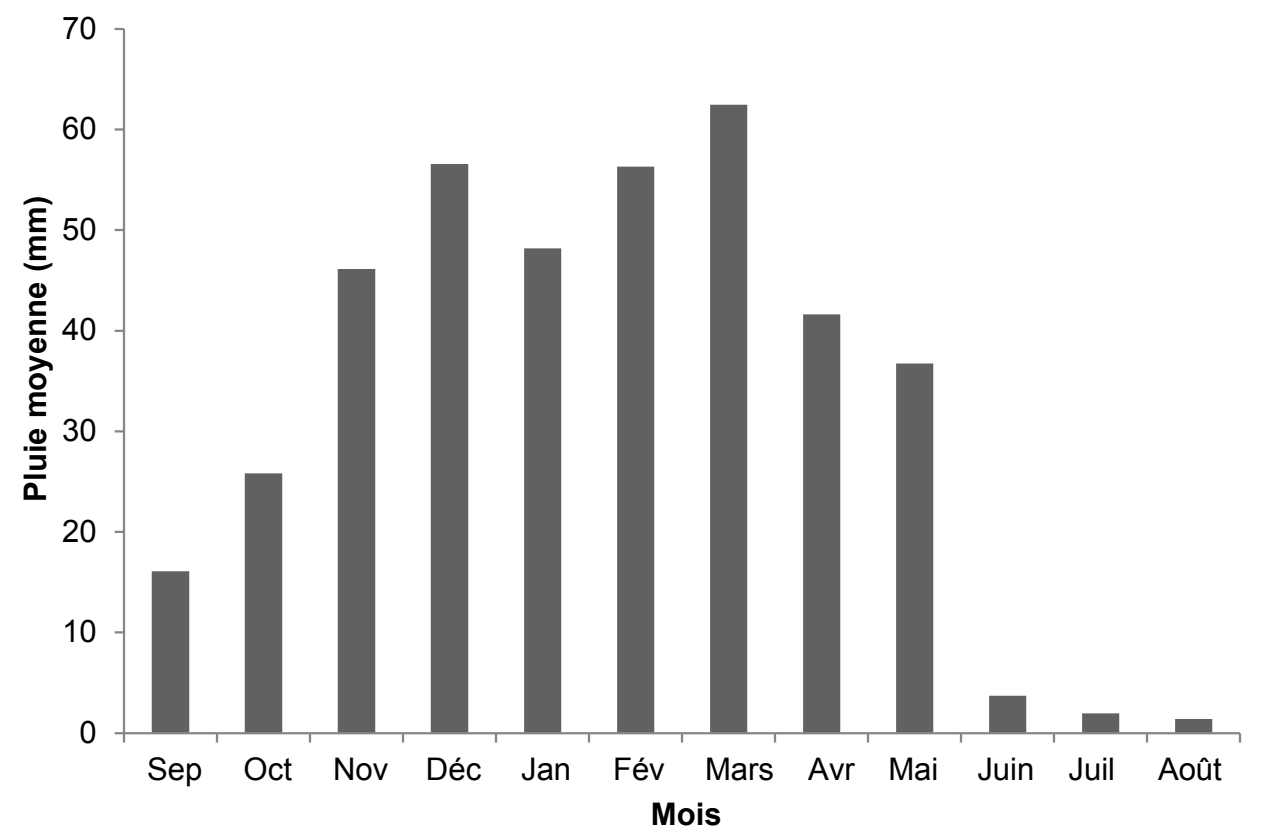

Figure 3. Répartition mensuelle moyenne de la pluie dans le bassin de l'oued Boumessaoud : station d'Henaya (1973-2010).

Average monthly rain assessment in the watershed of wadi Boumessaoud: Henaya Station (1973-2010).

récupérés. La filtration de l'eau en vue de recueillir les matières en suspension est réalisée sur du papier-filtre de type Whatman (porosité du filtre égale à $10 \mu \mathrm{m}$, temps de filtration $t=10,5 \mathrm{~s}$ ). Les filtres sont ensuite séchés à l'étuve. Ramenée à l'unité de volume $(1 \mathrm{~L})$ cette charge solide calculée est attribuée à la concentration instantanée en matières en suspension véhiculées par le cours d'eau en g. $\mathrm{L}^{-1}$. La fréquence des prélèvements est adaptée au régime hydrologique du cours d'eau, soit une mesure quotidienne en période d'écoulement normal caractérisée par des concentrations relativement faibles ou ne variant que très lentement au cours des $24 \mathrm{~h}$; en revanche les prélèvements d'eau sont intensifiés lors des événements averse-crue avec des prises à toutes les 30 min en fonction de l'amplitude des débits liquides. La méthode de travail établie consiste tout d'abord à calculer les apports liquides, solides et la dégradation spécifique au niveau du bassin d'étude. Le flux annuel des matières solides $A_{S}(\mathrm{t})$ en suspension exporté par l'oued Boumessaoud est donné par la formule : 


$$
A_{s}=\sum_{j=0}^{N} \frac{\left[\left(Q_{j+1} \cdot C_{j+1}\right)+\left(Q_{j} \cdot C_{j}\right)\right]}{2}\left(t_{j+1}-t_{j}\right)
$$

où $C_{j}$ et $C_{j+1}$ sont les concentrations relevées aux instants $t_{j}$ et $t_{j+1}$ correspondant respectivement aux débits liquide $Q_{j}$ et $Q_{j+1}^{j} ; \stackrel{N+1}{N}$ est le nombre de prélèvements effectués sur l'année considérée; $t_{j+1}-t_{j}$ est le pas de temps séparant deux prélèvements consécutifs.

De même, l'apport liquide annuel $A_{L}\left(\mathrm{~m}^{3}\right)$, engendrant le flux $A_{S}$ est calculé par la formule :

$$
A_{L}=\sum_{j=0}^{N} \frac{\left(Q_{j+1}+Q_{j}\right)}{2}\left(t_{j+1}-t_{j}\right)
$$

\section{RÉSULTATS ET DISCUSSION}

\subsection{Variabilité annuelle des apports liquides et solides}

L'apport liquide moyen annuel véhiculé par l'oued Boumessaoud a été évalué à $7,95 \mathrm{hm}^{3}$; il a engendré un apport solide moyen de $23851 \mathrm{t}$ soit une dégradation spécifique de $518 \mathrm{t} \cdot \mathrm{km}^{-2} \cdot \mathrm{an}^{-1}$. Les dégradations spécifiques ont subi une irrégularité importante $(\mathrm{CV}=0,79)$ et cela est dû à la variabilité spatio-temporelle des précipitations. Avec une concentration moyenne annuelle de 6,7 g. $\mathrm{L}^{-1}$, l'année 1990/91 a été celle qui a charrié le plus grand apport solide de toute la série d'étude (Figure 4), soit $20 \%$ de la charge globale. La dégradation spécifique est de $1550 \mathrm{t} \cdot \mathrm{km}^{-2} \cdot \mathrm{an}^{-1}$ soit trois fois la valeur moyenne annuelle de la période (septembre 1988 - août 2004). En effet, durant cette année hydrologique la crue survenue le 14 mars 1991 a été la plus exceptionnelle avec un débit spécifique de pointe atteignant $1,74 \mathrm{~m}^{3} \cdot \mathrm{s}^{-1} \cdot \mathrm{km}^{-2}$ et un débit solide correspondant à $0,025 \mathrm{t} \cdot \mathrm{s}^{-1} \cdot \mathrm{km}^{-2}$. Elle a drainé à elle seule un apport liquide considérable de $9 \mathrm{hm}^{3}$ représentant $68 \% \mathrm{du}$ volume global écoulé la même année. L’apport solide engendré lors de cet événement était de 70660 t soit une contribution de $95 \%$ du flux total annuel en sédiments. Toutefois, il est à noter que l'année hydrologique 1994/95 jugée par son apport liquide considérable de $13,10 \mathrm{hm}^{3}$, a enregistré un faible apport solide soit une contribution de l'ordre de $2 \%$. Ce résultat semble être dû à un ensemble de facteurs hydroclimatiques qui génèrent l'effet érosif des crues ainsi que leur période d'occurrence. La valeur de la dégradation spécifique trouvée pour notre bassin est proche de celles estimées par beaucoup d'auteurs qui ont travaillé sur des bassins de la région sud de la Méditerranée, en l'occurrence des taux d'érosion allant de 269 à $2569 \mathrm{t} \cdot \mathrm{km}^{-2} \cdot \mathrm{an}^{-1}$ proposés pour le Maghreb (HEUSCH et MILLIES-LACROIX, 1971), des valeurs de dégradations variant de 240 à $5900 \mathrm{t} \cdot \mathrm{km}^{-2} \cdot \mathrm{an}^{-1}$ (LAHLOU, 1994) au niveau des bassins du Maroc, ou bien encore des estimations entre 165 et $938 \mathrm{t} \cdot \mathrm{km}^{-2} \cdot \mathrm{an}^{-1}$ sur les bassins du Mouillah, de Sebdou, de l'Isser et de Sikak de l'Ouest algérien (BOUANANI, 2004).

\subsection{Variabilité intra-annuelle des apports liquides et solides}

Les variations des apports liquides moyens mensuels et les charges solides en suspension qui leur correspondent donnent un aperçu quant à la tendance globale de la susceptibilité du bassin versant à la production des sédiments (Figure 5). Deux phases sont à distinguer : une phase de grande mobilisation et entraînement de sédiments qui s'étale du mois d'octobre jusqu'au mois de mai soit une contribution qui s'élève à $96 \%$ de la charge moyenne annuelle. Une autre phase pauvre en perte en terre durant la saison estivale chaude et sèche dont les effets hydromorphologiques et climatiques se prolongent jusqu'au mois de septembre. En effet, dans la première phase on enregistre une pluviométrie importante durant l'hiver et le printemps, d'autre part les coefficients mensuels de débits (rapport du débit moyen mensuel au module interannuel de la période considérée [CMD]) et les coefficients d'écoulements (rapport entre la lame d'eau écoulée et la hauteur d'eau précipitée correspondante dans le bassin en $\mathrm{mm}\left[C_{e}\right]$ ) sont élevés plus particulièrement au mois d'octobre et mars avec respectivement 1,19 et 2,96 pour le CMD ainsi que $45 \%$ et $64 \%$ pour le $C_{\dot{e}}$.

Selon la figure 6 le printemps est la première saison de l'érosion dans le bassin versant d'étude avec un apport solide de 10000 t soit $43 \%$ de la charge globale, pour un apport liquide aussi important de $3,6 \mathrm{hm}^{3}$ soit $45 \%$ du volume total annuel écoulé. Les sapements de berges et des différents mouvements de masse des bas-versants qui atteignent les limites de liquidité en fin de printemps en sont la principale cause. En effet, les pluies du printemps, assez abondantes, survenues après un hiver pluvieux et froid avec une succession de gels et dégels favorisant la déstabilisation de la structure du sol et le rendant plus vulnérable à l'érosion, trouvent un sol meuble et déclenchent alors des écoulements fortement chargés. La succession des crues générées durant cette période de l’année a charrié la quasi-totalité des sédiments en suspension soit une contribution de $97 \%$. À l'automne, avec $30 \%$ de transport solide, l'impact des crues a été considérable totalisant une part de $71 \%$ du flux de sédiments transportés durant cette saison. Les quantités de sédiments évacuées par l'écoulement de l'oued Boumessaoud à ce moment de l'année sont influencées par les phénomènes de glaçage et de splash provoqués par les premières averses violentes de l'automne. Les crues qui surviennent en hiver n'ont pas un impact érosif comparable à celui des deux autres saisons, cela s'explique par le fait que des quantités importantes de particules solides ont été transportées par les premières crues d'automne (ACHITE et MEDDI, 2005; STEPHEN, 2000). En revanche, elles contribuent beaucoup 


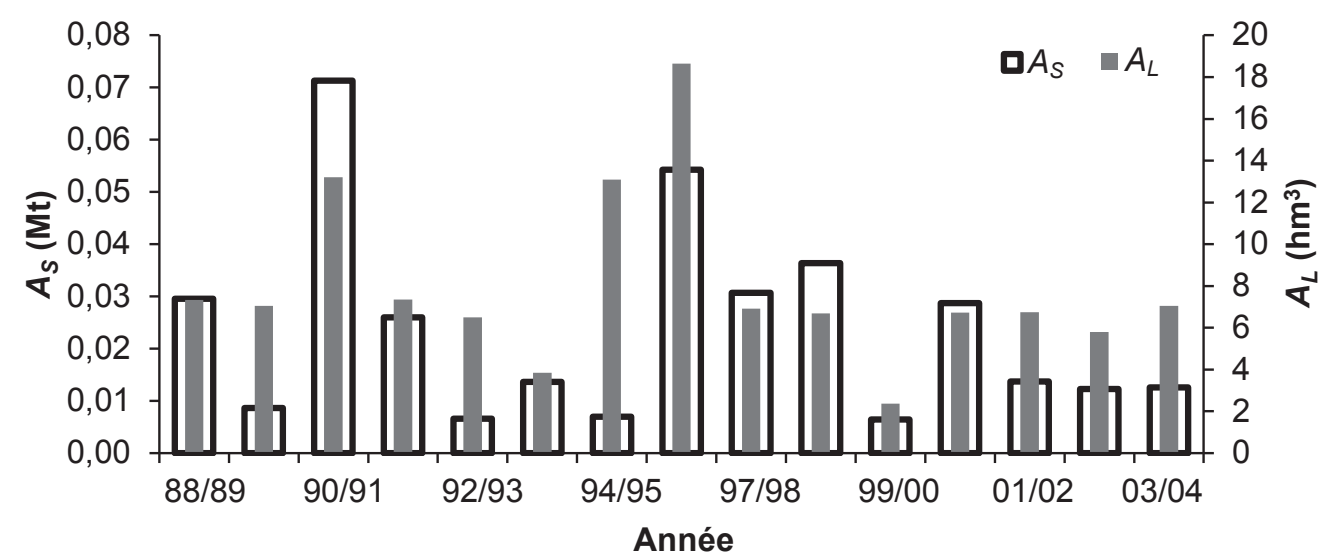

Figure 4. Variation interannuelle des apports solides et liquides de l'oued Boumessaoud : station d'Henaya (1988-2004).

Interannual variation of liquid and solid contribution of wadi Boumessaoud: Henaya Station (1988-2004).

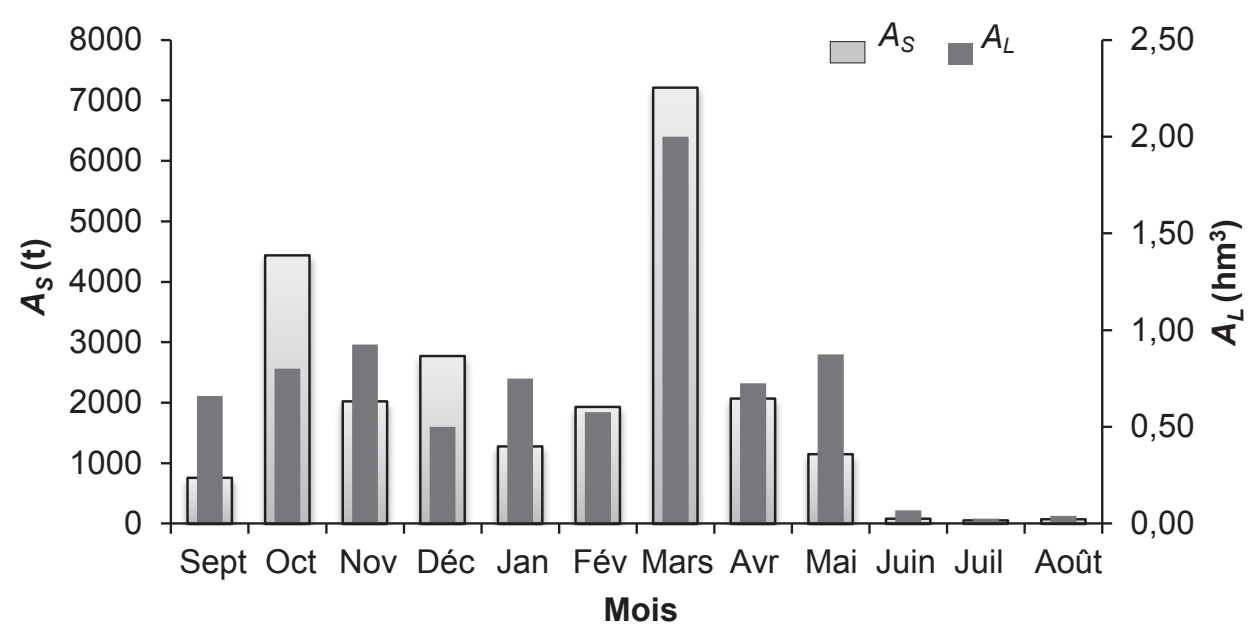

Figure 5. Variabilité mensuelle des apports solides et liquides dans l'oued Boumessaoud : station d'Henaya (1988-2004).

Monthly liquid and solid contribution in the wadi Boumessaoud: Henaya Station (1988-2004).

a

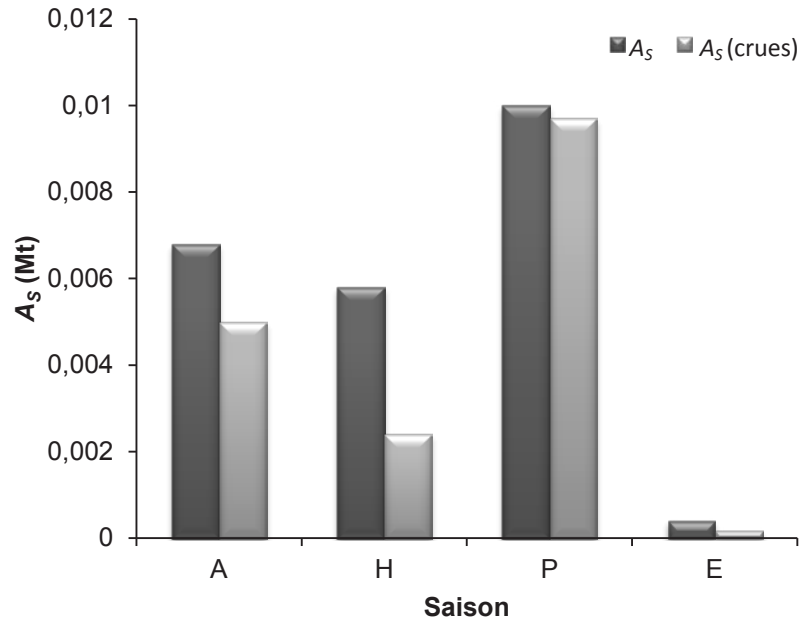

b

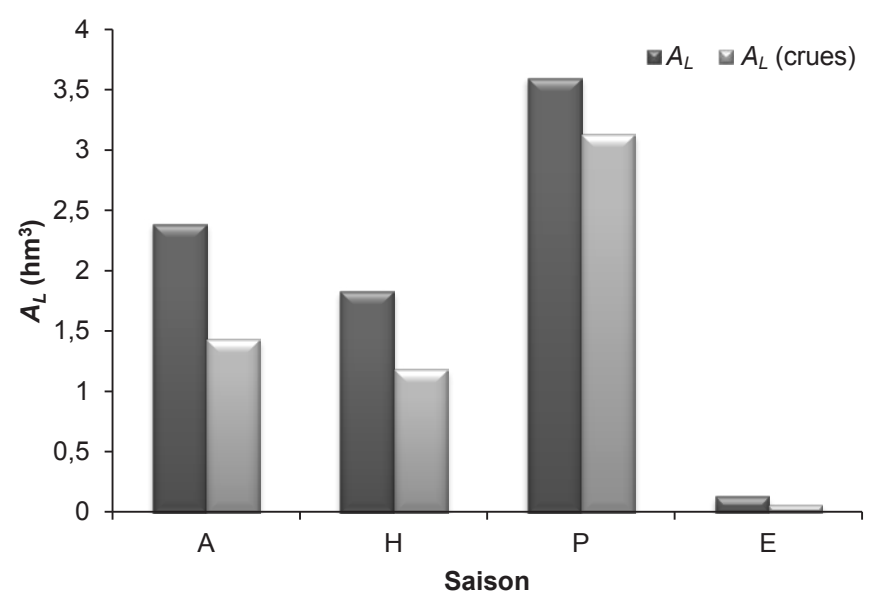

Figure 6. Apports saisonniers de l'oued Boumessaoud (1988/89-2003/04) : a) apports solides, b) apports liquides. A : automne; H : hiver; P : printemps; $\mathbf{E}$ : été.

Seasonal contribution of the wadi Boumessaoud (1988/89-2003/04): a) liquid contribution; b) solid contribution. 
plus à la reconstitution des nappes aquifères et participent au soutien des écoulements de surface jusqu'au mois de juin. L'été reste une saison sèche avec un transport quasi nul (environ $1 \%$ du flux global annuel).

\section{MODÉLISATION DU TRANSPORT SOLIDE}

Pour tenter de montrer à partir des mesures in situ la relation entre débits liquides et débits solides, une approche basée sur des statistiques des échantillons a été élaborée. Parmi les méthodes de contrôle des données $\left(Q_{L}, Q_{S}\right)$, nous avons les méthodes de corrélation - régression (BOIS et al., 2007). Le débit solide et le débit liquide évoluent en général suivant un modèle de puissance selon une relation empirique $Q_{S}=a Q_{L}^{b}$ communément appelée courbe du transport solide (CRAWFORD, 1991). Le tableau 1 résume les différents modèles statistiques retenus pour chaque groupement de données. Le choix du modèle est basé sur la valeur la plus élevée du coefficient de corrélation $R$ qui mesure l'éloignement des points expérimentaux de la droite de régression. Ce coefficient est calculé par la méthode des moindres carrés en faisant optimiser la somme des carrés des résidus (BOIS et al., 2007). Une première observation (Figure 7a) laisse découvrir qu’à de faibles débits liquides (moins de $0,022 \mathrm{~m}^{3} \cdot \mathrm{s}^{-1} \cdot \mathrm{km}^{-2}$ ) peuvent être associés des débits solides spécifiques assez importants (près de $0,217 \mathrm{~kg} \cdot \mathrm{s}^{-1} \cdot \mathrm{km}^{-2}$ ) déclenchés sûrement suite à des orages violents. On notera qu’à l'exception de la saison d'été (Figure 7e) où l'écoulement est quasi nul, les valeurs de l'exposant $\mathrm{b}$ de nos modèles varient entre 1,40 et 1,70 (Figure 7b, c, d et f) et sont proches de celles trouvées par bon nombre de chercheurs ayant travaillé sur des régions semi-arides (ACHITE, 2002; BOUANANI, 2004; MEGNOUNIF, 2007).

\subsection{Estimation de la dégradation spécifique à la station de Zénata}

\subsubsection{Validation des modèles utilisés à la station d'Henaya}

L'évaluation d'un modèle consiste à comparer les valeurs simulées aux valeurs observées. Des critères statistiques objectifs sont utilisés pour caractériser globalement la qualité de l'estimation. La série d'observations a été divisée en une période de calage (1988-1999) et une période de validation (2000-2004) où les modèles sont appliqués avec les paramètres estimés durant la période de calage, pour tester ainsi la performance du modèle dans l'estimation de la charge annuelle. Parmi les paramètres de choix d'un modèle adéquat, les plus utilisés sont :
- Coefficient de corrélation linéaire de Pearson $R$ : très utilisé comme premier indicateur de la qualité d'ajustement des points par rapport à la droite de régression;

- Coefficient d'efficacité ou coefficient de Nash : il exprime en pourcentage le rapport entre l'erreur relative aux valeurs estimées et mesurées avec la fluctuation des valeurs observées par rapport à la moyenne;

- Erreur d'estimation $E$ : c'est un des paramètres qui sert à mesurer la fiabilité d'un modèle en termes de précision de l'estimation de la charge solide.

Les résultats de calage et validation adaptés à différentes échelles temporelles (interannuelle, saisonnières et période de crues) sont résumés dans le tableau 2. Les résultats montrent que les coefficients de corrélation dépassent globalement 0,80 aussi bien pour la période de calage que celle de validation, excepté la période de l'été et celle des crues. Les critères de Nash ne sont guère satisfaisants et ne dépassent pas 0,45 (dues certainement au manque de données inhérent à nos séries d'observations). Toutefois, les meilleurs résultats du critère de Nash $(0,87)$ sont obtenus pour le printemps. Quant au troisième critère, celui représenté par l'erreur d'estimation, on voit bien qu'à l'échelle saisonnière et celle des crues, cette erreur diminue en période de validation en ne dépassant pas $20 \%$. Notons bien qu'à travers cette analyse et tenant compte des contraintes d'échantillonnage qui affectent souvent la qualité d'estimation, la fiabilité d'un modèle ne peut être validée en associant les trois critères à la fois. En effet pour un même coefficient $R$ on peut avoir des valeurs du critère de Nash et de l'erreur différentes. Ainsi les deux critères $E$ et $R$ par leurs valeurs moyennement satisfaisantes en période de validation nous ont permis de justifier le choix de ces trois modèles pour leur application au niveau de la station de Zénata qui contrôle l'ensemble du bassin d'étude avec une superficie de $118 \mathrm{~km}^{2}$. Lobjectif principal est de prévoir le taux de dégradation à l'exutoire du bassin.

\subsubsection{Application des modèles à la station de Zénata}

Le modèle en puissance représente la tendance globale des bassins versants méditerranéens à climat semi-aride où les conditions géomorphométriques et hydroclimatiques sont semblables entre elles (ACHITE et MEDDI, 2005; BENKHALED et REMINI, 2003; BOUANANI, 2004; MEGUENNI et REMINI, 2008; TERFOUS et al., 2001; TOUAIBIA et al., 2001). L'utilisation de ce type de modèles au droit de la station hydrométrique de Zénata située à l'exutoire et dépourvue de données concernant les concentrations nous a paru convenable dans de telles conditions. Notons qu'à l'échelle logarithmique la régression liant le couple $\left(Q_{L}, Q_{S}\right)$ s'écrit :

$$
\log Q_{S}=a+b \log Q_{L}+\log \varepsilon
$$

où $\varepsilon$ représente l'erreur commise log-normalement distribuée et $a$ et $b$ sont les paramètres de la régression. 
Tableau 1. Résultats de l'analyse statistique de la relation $Q_{S}=f\left(Q_{L}\right)$.

Table 1. Statistical analysis results of the relationship $Q_{S}=f\left(Q_{L}\right)$.

\begin{tabular}{lccc}
\hline Échelle temporelle & $\begin{array}{c}\text { Nombre } \\
\text { d'observations }\end{array}$ & $\begin{array}{c}\text { Coefficient de } \\
\text { corrélation }\end{array}$ & Modèle retenu \\
\hline Série complète & 1310 & 0,83 & $Q_{S}=1,59 Q_{L^{1,64}}$ \\
Automne & 263 & 0,84 & $Q_{S}=2,95 Q_{L^{1,69}}$ \\
Hiver & 547 & 0,83 & $Q_{S}=1,60 Q_{L^{1,72}}$ \\
Printemps & 469 & 0,85 & $Q_{S}=1,27 Q_{L^{1,57}}$ \\
Été & 109 & 0,49 & $Q_{S}=0,48 Q_{L}{ }^{0,79}$ \\
Ensemble de crues & 144 & 0,60 & $Q_{S}=3,15 Q_{L^{1,41}}$ \\
\hline
\end{tabular}

Tableau 2. Résultats des critères statistiques d'adaptation des modèles.

Table 2. Statistical criteria results of models adjustment.

\begin{tabular}{lcccc}
\hline \multicolumn{1}{c}{ Période } & Échelle & $\boldsymbol{R}^{\mathrm{a}}$ & $\mathbf{N a s h}^{\mathrm{b}}$ & $\boldsymbol{E} \mathbf{( \% ) ^ { \mathrm { c } }}$ \\
\hline \multirow{4}{*}{ Calage } & Inter & 0,85 & 0,32 & 7 \\
$(1988-1999)$ & Automne & 0,77 & 0,08 & -50 \\
& Hiver & 0,83 & 0,36 & -5 \\
& Printemps & 0,85 & 0,87 & -37 \\
& Été & 0,51 & 0,12 & 42 \\
& Crue & 0,58 & 0,72 & 56 \\
Validation & & & & \\
$(2000-2004)$ & Inter & 0,84 & 0,46 & 54 \\
& Automne & 0,9 & 0,36 & -17 \\
& Hiver & 0,83 & 0,71 & -18 \\
& Printemps & 0,81 & 0,30 & 5 \\
& Été & 0,57 & 0,015 & 10 \\
& Crue & 0,65 & 0,04 & -20 \\
\hline
\end{tabular}

${ }^{\mathrm{a}} R$ : coefficient de corrélation linéaire de Pearson;

$R=\frac{\sum_{i=1}^{n}\left(\log x_{i}-\overline{\log x}\right)\left(\log y_{i}-\overline{\log y}\right)}{\sqrt{\sum_{i=1}^{n}\left(\log x_{i}-\overline{\log x}\right)^{2}} \sqrt{\sum_{i=1}^{n}\left(\log y_{i}-\overline{\log y}\right)^{2}}}$ où $x_{i}$ : valeurs instantanées du débit

liquide; $y_{i}$ : valeurs instantanées du débit solide observé; $\bar{x}$ : moyenne des débits liquides;

$\bar{y}$ : moyenne des débits solides observés.

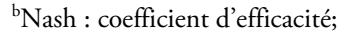

Nash $=1-\frac{\sum_{i=1}^{n}\left(y_{i}-y_{i a l}\right)^{2}}{\sum_{i=1}^{n}\left(y_{i}-\bar{y}\right)^{2}}$ où $y_{i c a l}$ : valeurs instantanées du débit solide calculé par les modèles.

${ }^{c} E$ : erreur d'estimation relative; $E(\%)=\frac{\left(y_{\text {iat }}-y_{i}\right)}{y_{i}} * 100$

Après retransformation à l'échelle arithmétique, cette relation s'écrit :

$$
Q_{S}=a Q_{L}^{b} \varepsilon
$$

où $\varepsilon$ est normalement distribuée.

Cette distribution différente entre l'écriture à l'échelle logarithmique et celle obtenue après retransformation est responsable de l'erreur ou biais donné par le modèle $(\mathrm{COHN}$ et al., 1989). Les résultats de cette quantification sont résumés au tableau 3. Les trois modèles testés pour leur application aux données de débits journaliers disponibles à la station de Zénata ont dégagé des dégradations de 858, 1110 et $541 \mathrm{t} \cdot \mathrm{km}^{-2} \cdot \mathrm{an}^{-1}$ pour les modèles annuels, saisonniers et des crues respectivement. Ces dégradations se situent dans la fourchette de celles estimées au niveau des autres bassins de la Tafna (GHENIM, 2001). Elles témoignent en effet d'une activité érosive de perte en terres due aux caractéristiques hydromorphométriques du bassin versant. 

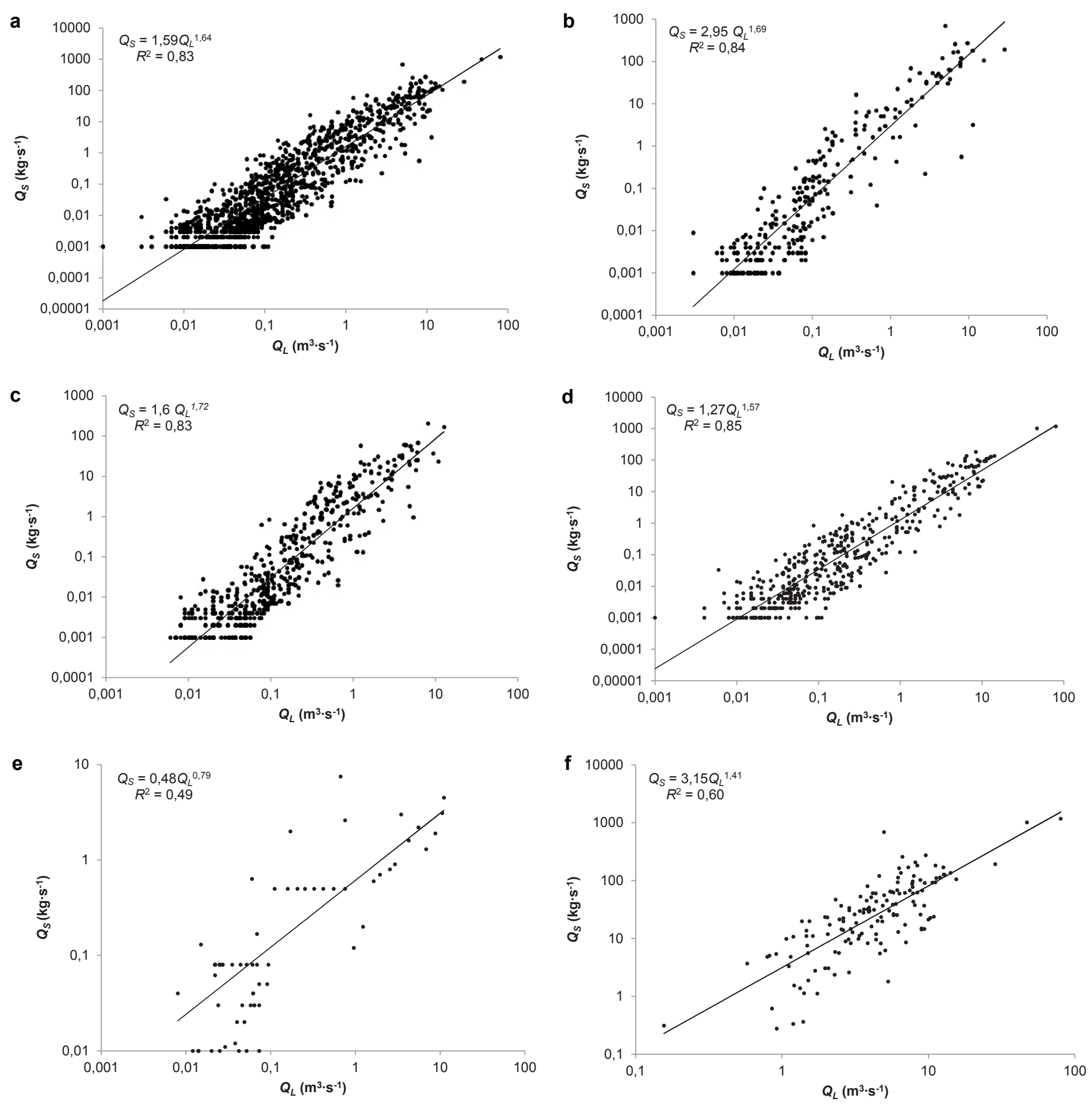

Figure 7. Relation entre les débits liquides et solides instantanés à différentes échelles temporelles : a) série complète; b) automne; c) hiver; d) printemps; e) été; f) ensemble de crues.

Relationship between instantaneous sediment yield and water discharges at different temporal scales: a) total series; b) Autumn; c) Winter; d) Spring; e) Summer; f) flood set. 
Tableau 3. Apport solide et dégradation spécifique dans le bassin versant de l'oued Boumessaoud : station de Zénata (1988-2004).

Table 3. Solid contribution and specific degradation in the watershed of wadi Boumessaoud: Zénata Station (1988-2004).

\begin{tabular}{|c|c|c|c|c|c|c|}
\hline Périodes & Annuel & Automne & Hiver & Printemps & Été & Période crue \\
\hline Modèle retenu & $1,59 Q_{L}^{1.64}$ & $2,95 Q_{L}^{1,69}$ & $1,60 Q_{L}^{1,72}$ & $1,27 Q_{L}^{1,57}$ & $0,48 Q_{L}^{0,79}$ & $3,15 Q_{L}^{1,41}$ \\
\hline Biais : $\varepsilon$ & 3,98 & 4,07 & 3,63 & 3,72 & 4,9 & 2,57 \\
\hline Modèle après retransformation & $6,33 Q_{L^{1.64}}$ & $12,02 Q_{L^{1,69}}$ & $5,81 Q_{L}^{1,72}$ & $4,72 Q_{L}^{1,57}$ & $2,35 Q_{L}^{0,79}$ & $8,1 Q_{L}^{1,41}$ \\
\hline Débit liquide spécif. moyen $\left(\mathrm{m}^{3} \cdot \mathrm{h}^{-1} \cdot \mathrm{km}^{-2}\right)$ & 20,16 & 20,88 & 10,80 & 46,80 & 2,16 & 122,4 \\
\hline Débit solide spécif. moyen $\left(\mathrm{kg} \cdot \mathrm{h}^{-1} \cdot \mathrm{km}^{-2}\right)$ & 97,9 & 193,2 & 29,7 & 281,9 & 8,85 & 1752,44 \\
\hline Apport solide/période $(\mathrm{t})$ & 101187 & 49241 & 7572 & 71852 & 2256 & 958300 \\
\hline Apport solide annuel (t) & 101187 & \multicolumn{4}{|c|}{130922} & 63886 \\
\hline Dégradation spécif. $\left(\mathrm{t} \cdot \mathrm{km}^{-2} \cdot \mathrm{an}^{-1}\right)$ & 858 & \multicolumn{4}{|c|}{1110} & 541 \\
\hline
\end{tabular}

5. ÉVOLUTION DELA CONCENTRATION DES SÉDIMENTS EN SUSPENSION EN FONCTION DES DÉBITS LIQUIDES DURANT LES PÉRIODES DE CRUES

L'analyse de la relation qui relie la concentration des sédiments en suspension $\left(C_{S}\right)$ et le débit liquide $\left(Q_{L}\right)$ à l'échelle d'un événement hydrologique de crue a énormément contribué à la compréhension du phénomène du transport des sédiments à travers le système de drainage d'un bassin versant (DICKINSON et BOLTON, 1992; HEIDEL, 1956; IRVINE et DRAKE, 1987; PEART et WALLING, 1982). La présente étude de l'hystérésis est basée sur les travaux de WILLIAMS (1989). L'auteur propose une classification de ces relations basées sur le rapport $C_{S} / Q_{L}$ durant les phases de montée de crue et de décrue. Cinq modèles de relations sont ainsi établis. Leur illustration graphique ayant été présentée dans divers articles, nous nous contenterons de montrer la signification des différentes formes de courbes $C_{S}=f\left(Q_{L}\right)$ ci-dessous qui se sont manifestés lors des événements majeurs :

- Forme I : courbe simple de type linéaire;

- Forme II : boucle dans le sens des aiguilles d'une montre dite hystérésis positive ou encore appelée " hystérésis rétrograde ";

- Forme III : boucle dans le sens contraire des aiguilles d'une montre dite hystérésis négative ou appelée aussi « hystérésis orthograde ";

- Forme en huit : combinaison des deux formes II et III.

Les crues prises en considération sont celles qui se manifestent par une variation notable du débit liquide. D'après REMENIERAS (1976), les crues sont des débits égaux ou supérieurs à un certain multiple du module annuel (3 à 5 fois le débit annuel). Ce critère ainsi adopté nous a permis de sélectionner 22 crues au total pour notre période d'observation. Lapproche par les hystérésis appliquée aux crues survenues au niveau du bassin d'étude a fait ressortir les différentes classes d'évolution de cette relation $C_{S}=f\left(Q_{L}\right)$ (Tableau 4). Celuici montre que $50,5 \%$ des crues ayant un impact érosif ont manifesté une forme en II, soit un maximum de charge solide atteint bien avant celui du débit. Ce décalage est lié à un épuisement du stock de sédiments disponible avant le maximum du débit (WALLING, 1984; WOOD, 1977) comme il a été constaté en automne durant la crue du 22 novembre 1988 très importante par sa plus forte concentration $\left(138 \mathrm{~g} \cdot \mathrm{L}^{-1}\right)$ de toute la série d'observations (Figure $8 \mathrm{a}$ et b). La forme II résulte aussi d'une activité érosive de remobilisation de particules fines (KATTAN et al., 1987) ou d'une remise en suspension (ARNBORG et al.,1967) comme, par exemple, la crue du 20 mars 1989 ou celle du 14 janvier 1990. La représentation de quelques caractéristiques des 22 crues enregistrées a été illustrée au tableau 5.

Le printemps avec $23 \%$ de forme II a été la saison d'écoulement abondant avec des taux interannuels moyens de $64 \%$ au mois de mars et $52 \%$ au mois de mai. Cela montre davantage une concentration des crues dans cette saison. Leur succession en nombre important permet d'affirmer une activité érosive de remobilisation de particules fines pour de faibles débits le long du lit fluvial et sur les terrasses latérales. L'hiver a été marqué par la même fréquence d'hystérésis positive que le printemps. En effet, cette saison a connu une pluviométrie élevée d'une valeur moyenne de $161 \mathrm{~mm}$ soit $37 \%$ du total moyen interannuel. L'effet donc des averses sur le bassin versant après une longue phase de dessèchement très dure facilite l'épuisement de stock de sédiments disponibles avant le maximum du débit. Les apports solides ont connu une diminution sensible, car la plus grande partie des particules solides a été transportée par les premières crues d'automne (ACHITE et MEDDI, 2005; STEPHEN, 2000).

La forme III (Figure 8c et d), très fréquente au printemps, résulte du fait que le sol étant plus ou moins saturé et couvert de végétation, il résiste mieux à la pluie. Lérosion et le transport solide se verront légèrement retardés par rapport aux écoulements. Ainsi, ce n'est qu'après une érosion prolongée que la concentration en matières en suspension augmente, 
Tableau 4. Fréquences des classes d'hystérésis au niveau du bassin versant de l'oued Boumessaoud : station d'Henaya (1988/89-2003/04).

Table 4. Frequency of hysteresis classes in the watershed of wadi Boumessaoud: Henaya Station (1988/89-2003/04).

\begin{tabular}{lccccc}
\hline Saison & $\begin{array}{c}\text { Nombre de } \\
\text { crues }\end{array}$ & $\begin{array}{c}\text { Forme I } \\
\mathbf{( \% )}\end{array}$ & $\begin{array}{c}\text { Forme II } \\
\mathbf{( \% )}\end{array}$ & $\begin{array}{c}\text { Forme III } \\
\mathbf{( \% )}\end{array}$ & $\begin{array}{c}\text { Forme (huit) } \\
\mathbf{( \% )}\end{array}$ \\
\hline Automne & 5 & 4,5 & 4,5 & 4,5 & 9 \\
Hiver & 7 & 4,5 & 23 & 4,5 & - \\
Printemps & 10 & 4,5 & 23 & 18 & - \\
\hline
\end{tabular}
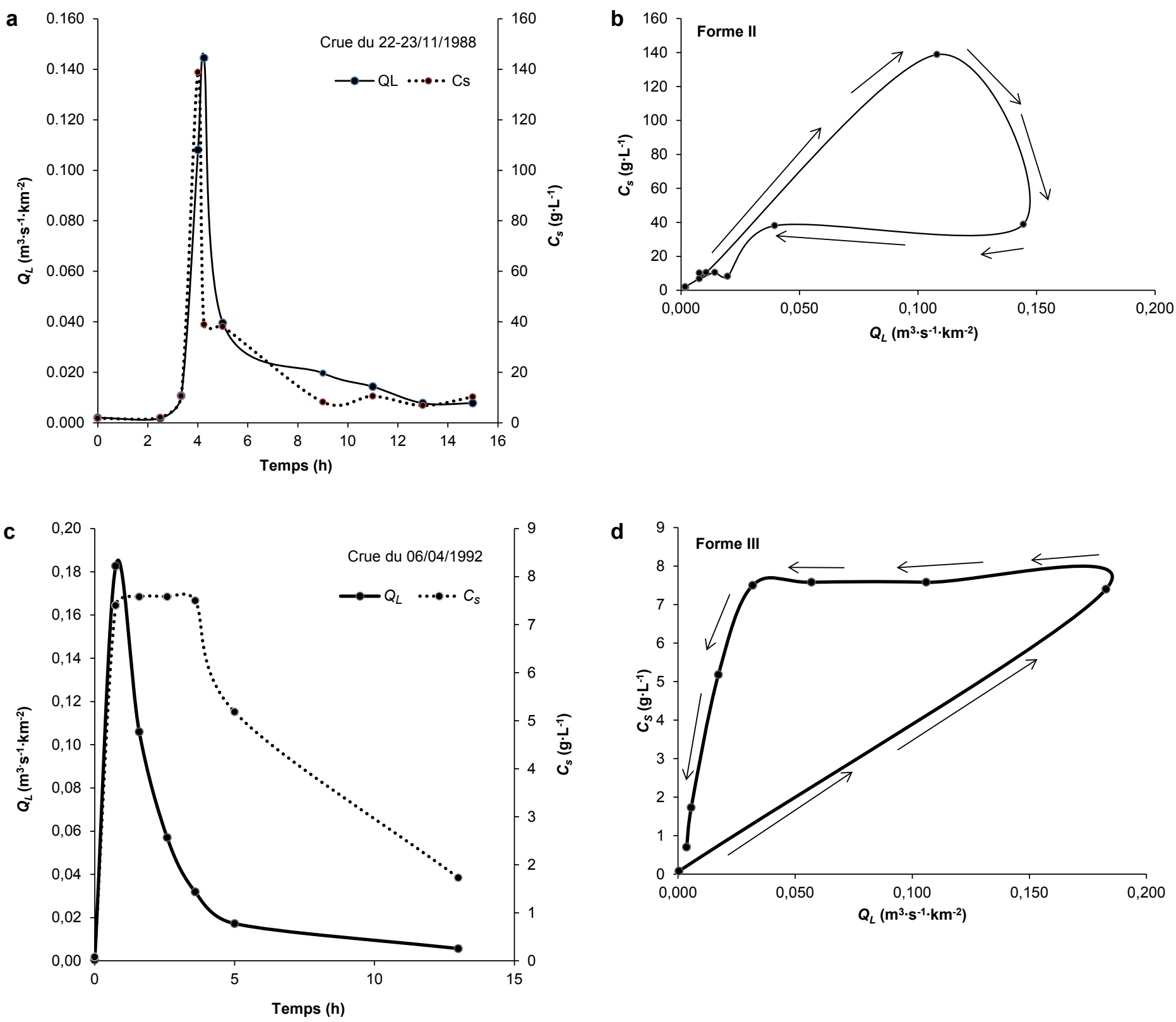

Figure 8. Graphes temporels de la concentration des sédiments $\left(C_{S}\right)$, du débit liquide $\left(Q_{L}\right)$ et du type d'hystérésis du bassin versant de l'oued Boumessaoud (station d'Henaya) : a) et b) crue du 22 au 23/11/1988; c) et d) crue du 06/04/1992.

Temporal graph of the concentration of sediment (CS), water discharge (QL) and hysteresis pattern during flood events in the wadi Boumessaoud at Henaya station: a) and b) flood of 22 to 23/11/88; c) and d) flood of 06/04/1992. 

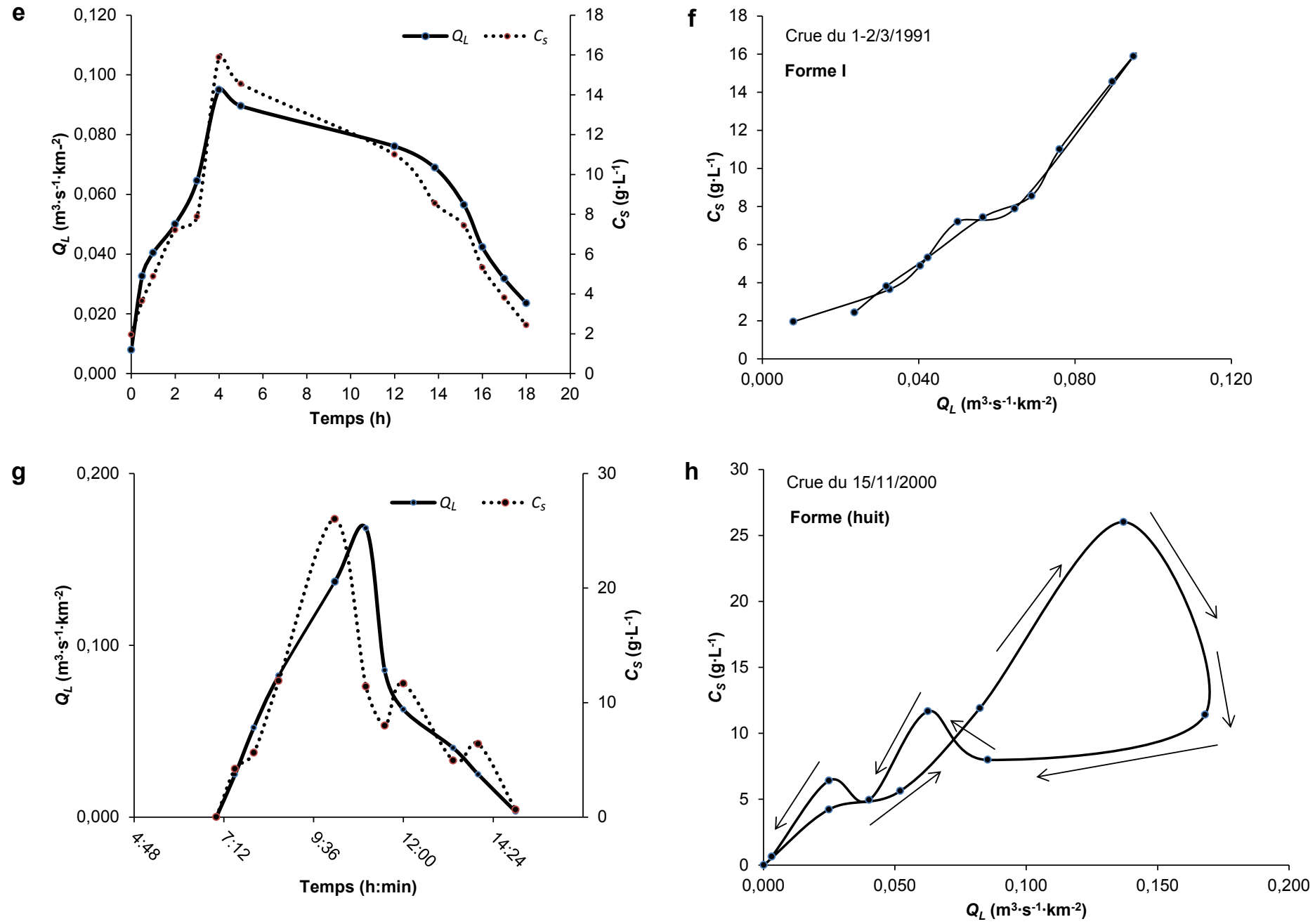

Figure 8. Graphes temporels de la concentration des sédiments $\left(C_{S}\right)$, du débit liquide $\left(Q_{L}\right)$ et du type d'hystérésis du bassin versant de l'oued (suite) Boumessaoud (station d'Henaya) : e) et f) crue du 1-2/3/1991; g) et h) crue du 15/11/2000.

(continued) Temporal graph of the concentration of sediment $\left(C_{S}\right)$, water discharge $\left(Q_{L}\right)$ and hysteresis pattern during flood events in the wadi Boumessaoud at Henaya station: $e$ ) and f) flood of 1-2/03/1991; g) and h) flood of 15/11/2000.

ce qui donne un pic des concentrations retardé par rapport à celui des débits. Pour la forme I (Figure 8e et f) les sédiments sont en grande partie arrachés des berges et du lit du cours d'eau (MEGNOUNIF, 2007). La forme en huit (Figure 8g et h) survenue en automne permet de voir au départ que le pic des concentrations est atteint avant celui des débits, suite à la réserve de sédiments disponible après une longue période sèche. La tendance s'inverse avec le temps dès l'épuisement du stock de sédiments.

\section{RELATION ENTRE LES APPORTS SOLIDES DURANT LES CRUES ET LES SOURCES DES SÉDIMENTS TRANSPORTÉS}

La forme de l'hystérésis dépend de plusieurs facteurs comme la disponibilité des sédiments et leur distance à la station hydrométrique, l'intensité et la distribution des précipitations, l'abondance de l'écoulement, l'ampleur du ruissellement sur les versants et la saison d'occurrence de la crue.

\subsection{Relation en fonction du temps des valeurs $C_{S}$ max et $Q_{L}$ max pendant les crues}

En mettant en graphe les valeurs de $Q_{L}$ max et $C_{S} \max$ relatives aux 22 événements de crues recensées, on peut distinguer (Figure 9) que leur variation au cours des cycles hydrologiques est très contrastée. Quoique les concentrations maximales n'aient pas connu de fortes amplitudes dans leur variation, les apports solides restent particulièrement influencés par les valeurs maximales des concentrations de sédiments en suspension véhiculées et des débits liquides. Celles-ci se sont 
Tableau 5. Paramètres caractéristiques des 22 crues principales (1988-2004).

Table 5. Characteristic parameters of 22 main floods (1988-2004).

\begin{tabular}{|c|c|c|c|c|c|c|}
\hline Date & Forme & $\begin{array}{l}\text { Durée } \\
\text { (h) }\end{array}$ & $\begin{array}{c}Q_{L} \max ^{\mathrm{a}} \\
\left(\mathbf{m}^{3} \cdot \mathbf{s}^{-1} \cdot \mathbf{k m}^{-2}\right)\end{array}$ & $\begin{array}{c}\max C_{S-\max } Q_{L}^{\mathrm{b}} \\
(\mathrm{h})\end{array}$ & $\begin{array}{c}C_{S} \max ^{\mathrm{c}} \\
\left(\mathrm{g} \cdot \mathrm{L}^{-1}\right)\end{array}$ & $\begin{array}{c}A_{S}^{\mathrm{d}} \\
\left(10^{3} \mathrm{t} \cdot \mathrm{km}^{-2}\right)\end{array}$ \\
\hline $22 / 11 / 1988$ & II & 15 & 0,144 & 0,25 & 138,79 & 0,485 \\
\hline 27/09/1997 & III & 3 & 0,622 & 0,5 & 6,8 & 0,313 \\
\hline $14 / 10 / 2000$ & huit & 7,66 & 0,243 & 0,5 & 28,56 & 0,353 \\
\hline $15 / 11 / 2000$ & huit & 8,66 & 0,165 & 0,5 & 26,02 & 0,302 \\
\hline $25 / 11 / 2002$ & I & 1,16 & 0,17 & 0 & 12,36 & 0,268 \\
\hline 04/01/1990 & II & 13 & 0,113 & 1 & 14,56 & 0,132 \\
\hline $14 / 01 / 1990$ & II & 4,08 & 0,101 & 3,08 & 5,9 & 0,068 \\
\hline 20/01/1994 & II & 6,4 & 0,275 & 13,34 & 4,4 & 0,093 \\
\hline 18/01/1999 & II & 24 & 0,203 & 8,25 & 13,34 & 0,112 \\
\hline $30 / 12 / 1999$ & II & 9 & 0,03 & 1 & 1,04 & 0,023 \\
\hline $27 / 02 / 2001$ & III & 5,16 & 0,053 & 0,66 & 9,92 & 0,072 \\
\hline $10 / 12 / 2003$ & I & 16,5 & 0,177 & 0 & 25,48 & 0,274 \\
\hline 20/03/1989 & II & 36 & 0,308 & 11,5 & 20,96 & 0,623 \\
\hline $14 / 03 / 1991$ & II & 9,3 & 1,74 & 2,75 & 21,55 & 1,536 \\
\hline 05/03/1992 & III & 21 & 0,183 & 0 & 21,68 & 0,226 \\
\hline 06/04/1992 & III & 13 & 0,183 & 0,8 & 7,58 & 0,113 \\
\hline 09/04/1992 & III & 6,8 & 0,083 & 3 & 9,31 & 0,095 \\
\hline $04 / 05 / 1992$ & II & 10 & 0,217 & 1,75 & 9,1 & 0,142 \\
\hline 05/05/1993 & II & 22 & 0,204 & 3.5 & 11,84 & 0,210 \\
\hline 03/05/1998 & II & 7 & 0,115 & 1 & 2,18 & 0,068 \\
\hline 01/03/1999 & I & 11 & 0,134 & 0 & 15,14 & 0,336 \\
\hline $07 / 05 / 2002$ & III & 9 & 0,224 & 0,75 & 2,94 & 0,064 \\
\hline
\end{tabular}

${ }^{a} Q_{L} \max$ : débit liquide spécifique maximal atteint durant la crue;

${ }^{\mathrm{b}} \max C_{S}$-max $Q_{L}$ : écart de temps entre les deux pics atteints $Q_{L}$ max et $C_{S} \max$;

${ }^{\mathrm{C}} C_{S}$ max : concentration maximale de sédiment atteinte durant la crue;

${ }^{\mathrm{d}} A_{S}$ : apport solide spécifique durant toute la crue.

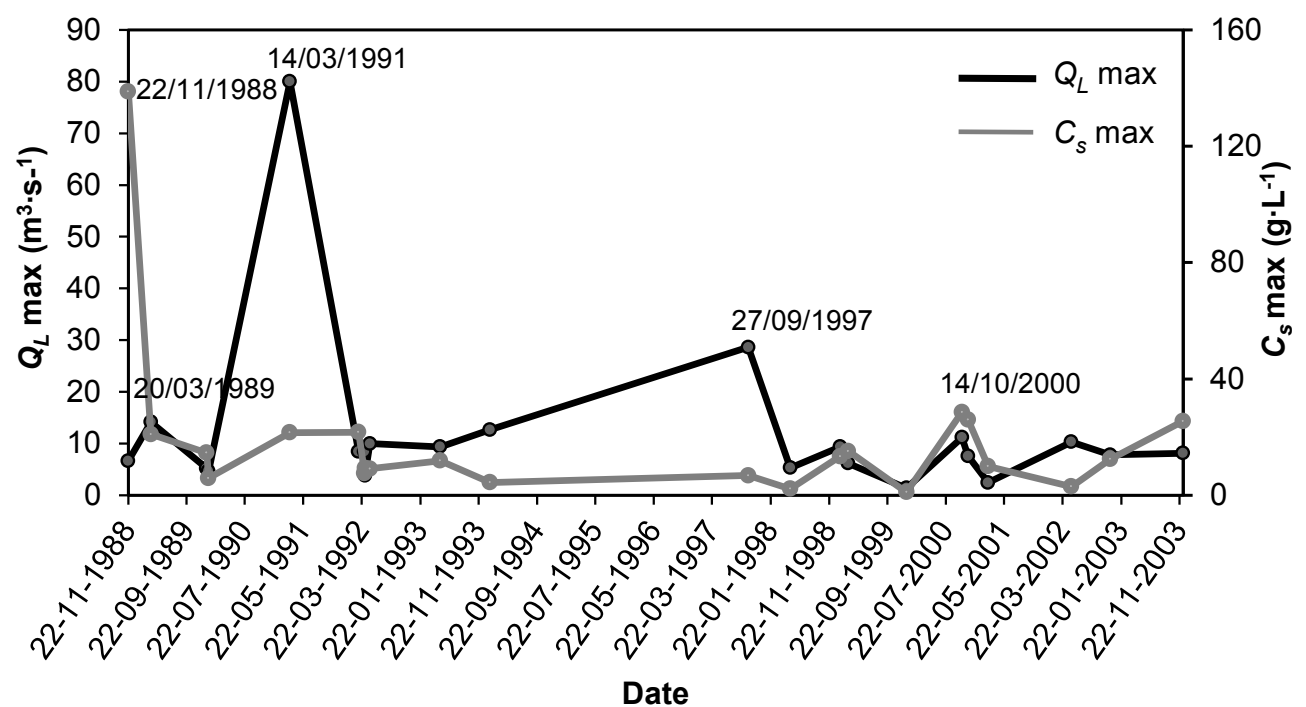

Figure 9 Variation de $C_{S} \max$ et $Q_{L} \max$ durant la période d'observation (1988-2004).

Change in $C_{S}$ max and $Q_{L}$ max during observation period (1988-2004). 
manifestées en automne comme la crue du 22 novembre 1988 avec un $C_{S}$ max avoisinant $138 \mathrm{~g} \cdot \mathrm{L}^{-1}$ et celle du 14 octobre 2000 ou au printemps comme la crue du 20 mars 1989 et surtout celle du 14 mars 1991 avec un $Q_{L} \max$ de $1,74 \mathrm{~m}^{3} \cdot \mathrm{s}^{-1} \cdot \mathrm{km}^{-2}$.

Ceci dit, la représentation des variations saisonnières des apports liquides $A_{L}$ et solides $A_{S}$ (Figure 10) durant les 22 crues laisse apparaître une prédominance de la saison du printemps sur les deux autres saisons tant en apport solide que liquide. Cette saison a favorisé la fourniture d'une grande quantité de sédiments de différentes sources en raison de la fréquence d'occurrence des crues égale à 45,5\%. L'automne, avec une fréquence des crues $(22,5 \%)$ inférieure à celle de l'hiver (32\%), présente un apport solide dépassant le double de celuici. C'est en effet la particularité de cette saison favorable à la combinaison des conditions hydrosédimentaires et climatiques pour le déclenchement du transport solide au sein du bassin versant. Le ruissellement sur les versants participe de manière significative à cette dynamique érosive des sédiments.

\subsection{Calcul des apports solides et sources des sédiments pendant les crues}

Les zones arides et semi-arides sont caractérisées par une agressivité du climat et spécifiquement à la saison de l'automne, celle-ci succédant à une longue période de préparation de matériaux allant de juin à septembre et pouvant même s'étendre jusqu'à l'hiver, comme c'est le cas de notre série d'observations. En effet, cette saison se caractérise par une multiplication des activités de labours sur les versants et tout près des rives de l'oued favorisant ainsi la production de matériaux en suspension provoqués par l'effet splash des pluies érosives et des eaux de ruissellement. Dans ces conditions, les sédiments seront d'autant plus disponibles sur les versants (pour la forme en huit) que dans le réseau de drainage (pour les formes II et III). Par ailleurs, pour les deux autres saisons, l'hiver et le printemps, les écoulements deviennent importants et les précipitations, par leur abondance, arrivent à mobiliser de grandes quantités de sédiments. Les sources sont multiples et l'on assiste à une alternance d'entraînement des particules sédimentaires depuis les versants, mais surtout du lit et des berges de l'oued (pour la forme II) (SMITH et DRAGOVITCH, 2009). La forme I sera régulée par une production continue de sédiments durant l'averse depuis le réseau de drainage. Étant donné le manque de données d'échantillonnage et leur mode de prélèvement toujours limité et incomplet, les sources de sédiments pendant les crues et leur disponibilité avant d'arriver à la station de jaugeage sont influencées par beaucoup d'autres paramètres. En plus de ceux cités ci-dessus on souligne l'effet de l'état hydrique du sol et la cohésion des particules qui diffère d'une saison à une autre ainsi que l'amplitude du débit et de la concentration aussi bien en montée de la crue qu’à la décrue.
Sans être trop exhaustive, cette approche s'inspire de celles déjà avancées (ETCHANCHU et PROBST, 1986; MEGNOUNIF, 2007; MEGNOUNIF et al., 2013) et cherche à quantifier et identifier les sources saisonnières des sédiments durant la période des 22 crues en décomposant le solidogramme (graphique donnant la variation du débit solide au cours de la crue) de chacune des crues pour déterminer la part des sédiments provenant des versants et ceux résultant du réseau hydrographique et cela en se basant davantage sur les critères introduits précédemment.

Le tableau 6 résume les différentes contributions des versants et du réseau de drainage dans le transport des sédiments au cours des 22 crues recensées. Il ressort que les sédiments en automne sont beaucoup plus disponibles sur les versants $(20 \%)$ comparativement aux deux autres saisons et qu'au printemps la tendance s'inverse et c'est dans le fond du lit et des berges que l'écoulement puise l'essentiel des sédiments. Cela confirme le type de réaction des bassins versants soumis à des variables de forçage multiples en climat semi-aride contrairement aux bassins des zones tempérées et humides. Il faut noter que les résultats diffèrent d'une étude à une autre en raison de la disponibilité de données sur les événements exceptionnels et en raison aussi de la fiabilité des estimations sur le transport solide.

Au terme de cette partie, nous estimons avoir pu donner à l'échelle saisonnière, une notion approximative sur le rapport qui existe entre les sources de sédiments dans le bassin versant et leurs apports successifs pendant les 22 événements de crues durant la période d'observation (1988-2004), pour tenter ainsi d'expliquer la dynamique du phénomène de l'érosion qui reste très complexe et très aléatoire. On retient que les crues de la saison du printemps par leur importance et leur nombre mobilisent davantage des particules le long du réseau (45\%) et sur les versants (13\%) et contribuent à $58 \%$ de l'apport solide global.

\section{CONCLUSION}

La dégradation spécifique estimée pour le bassin d'oued Boumessaoud, contrôlée par la station de jaugeage d'Henaya a été de $518 \mathrm{t} \cdot \mathrm{km}^{-2} \cdot \mathrm{an}^{-1}$. Elle est élevée par rapport à celle du globe, l'Afrique et l'Europe. Elle se situe dans la fourchette de 269 à $2569 \mathrm{t} \cdot \mathrm{km}^{-2} \cdot \mathrm{an}^{-1}$ proposée pour le Maghreb (HEUSCH et MILLIES-LACROIX, 1971). Une forte variabilité interannuelle des apports solides a été constatée avec une capacité d'érosion qui diffère entre les périodes humides et sèches. À l'échelle saisonnière, les contributions enregistrées ont donné $31 \%$ pour l'automne, $25 \%$ pour l'hiver et $43 \%$ 


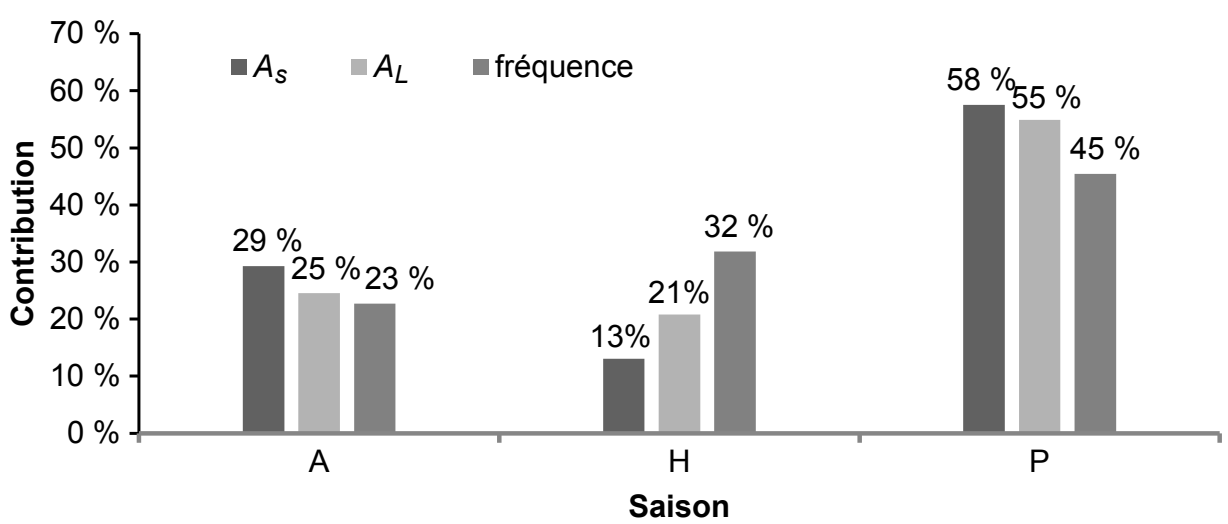

Figure 10 Contribution saisonnière des deux apports $A_{S}$ et $A_{L}$ durant les 22 crues. A : automne; $\mathrm{H}$ : hiver; $\mathbf{P}$ : printemps.

Seasonal contribution of $A_{S}$ and $A_{L}$ supplies during 22 flood events.

Tableau 6. Source de sédiments et leur contribution saisonnière. Table 6. Sediment source and their seasonal contribution.

\begin{tabular}{lccc}
\hline $\begin{array}{l}\text { Apport solide } \\
\text { saisonnier }\end{array}$ & Automne (\%) & Hiver (\%) & Printemps (\%) \\
\hline Des versants & 20 & 3 & 13 \\
$\begin{array}{l}\text { Du réseau } \\
\text { hydrographique }\end{array}$ & 9 & 10 & 45 \\
Total & 29 & 13 & 58 \\
\hline
\end{tabular}

pour le printemps. Le potentiel érosif est relativement élevé durant le printemps en raison des effondrements de berges et des mouvements de masses souvent déclenchés en cette saison. À l'échelle événementielle, on montre que les crues recensées durant la période d'étude (1988-2004) représentent en moyenne $10 \%$ du temps annuel. Elles contribuent à $57 \%$ en apport liquide sur un apport annuel moyen estimé à 7,95 millions de mètres cubes. Alors que le flux des matières solides transportées en suspension par ces dernières constitue en moyenne la quasi-totalité, soit $90 \%$ du flux moyen annuel évalué à 23851 t. La relation liant les débits liquides aux débits solides en suspension pour notre bassin d'étude est de type puissance $\left(Q_{S}=a Q_{L}^{b}\right)$. L'analyse de l'évolution des concentrations en fonction des débits liquides durant les crues montre que la réponse des concentrations aux débits suit quatre modèles de courbes : simple $(13,5 \%)$, dans le sens d'une aiguille d'une montre $(50,5 \%)$, dans le sens contraire des aiguilles d'une montre ( $27 \%)$ et forme en huit (9\%). Trois périodes distinctes se font remarquer par des comportements différents dans le fonctionnement du système. En automne, différents processus se conjuguent et favorisent la production des sédiments, essentiellement à partir des versants. Durant la période hiver et printemps, où l'on a enregistré le plus de crues (79\%), la production des sédiments a été relativement élevée. Elle s'effectue principalement dans le réseau hydrographique (lit, berges et affluents).
À la fin de cette étude et après avoir donné un aspect quantitatif du transport solide dans ce bassin, une réflexion à l'échelle du bassin versant est essentielle et indispensable pour réduire significativement les risques de l'érosion hydrique. Pour augmenter l'infiltration du sol et faciliter la sédimentation ou laminer les crues ou encore gérer les débits, la combinaison de certaines pratiques culturales (déchaumage, binage et écroûtage) et d'autres techniques d'aménagement (reboisement, bandes enherbées, talus, haies, fossés, etc.) est donc nécessaire pour lutter efficacement contre le ruissellement superficiel et contre l'érosion des terres surtout à vocation agricole.

\section{RÉFÉRENCES BIBLIOGRAPHIQUES}

ACHITE M. (2002). Approche statistique d'évaluation du transport solide dans le bassin versant de l'oued Mina (Nord-Ouest algérien). Watmed (Tunisie), 2, 894-899.

ACHITE M. et M. MEDDI (2004). Estimation du transport solide dans le bassin versant de l'oued Haddad (NordOuest algérien). Sécheresse, 15 (4), 367-373.

ACHITE M. et M. MEDDI (2005). Variabilité spatiotemporelle des apports liquide et solide en zone semi-aride. Cas du bassin de l'oued Mina (Nord-Ouest Algérien). Rev. Sci. Eau, 18, 37-56.

ARNBORG L., H.J. WALKER et J. PEIPPO (1967). Suspended load in the Colville River, Alaska, 1962. Geografiska Annale, Ser. A, 49 (2-4), 131-144. 
BENKHALED A. et B. REMINI (2003). Analyse de la relation de puissance : débit solide - débit liquide à l'échelle du bassin versant de l'oued Wahrane (Algérie). Rev. Sci. Eau, 16 (3), 333-356.

BOIS Ph., Ch. OBLED et I. ZIN (2007). Introduction au traitement de données en hydrologie. $7^{\mathrm{e}}$ édition revue, cours polycopié de l'ENSHMG (École Nationale Supérieure d'Hydraulique et de Mécanique de Grenoble), Grenoble, France, $265 \mathrm{p}$.

BOUANANI A. (2004). Hydrologie, transport solide et modélisation : étude de quelques sous-bassins de la Tafna (NW - Algérien). Thèse de doctorat, Univ. Tlemcen, Algérie, $250 \mathrm{p}$.

BOU KHEIR R., M.C. GIRARD, M. KHAWLIE et C. ABDALLAH (2001). Érosion hydrique des sols dans les milieux méditerranéens : une revue bibliographique. Etud. Gest. Sols, 8 (4), 231-245.

BOUROUBA M. (1998). Phénomène de transport solide dans les Hauts Plateaux orientaux. Cas de l'oued Logmane et oued Leham dans le bassin de la Hodna. Rev. Sci. Eau, 9, $5-11$.

COHN T.A., L.L. DELONG, E.J. GILROY, R.M. HIRSCH et D.K. WELLS (1989). Estimating constituent loads. Water Resour. Res., 25 (5), 937-947.

CRAWFORD C.G., (1991). Estimation of suspendedsediment rating curves and mean suspended sediment load. J. Hydrol., 129, 331-348.

DE PLOEY J., A. IMESON et L.R. OLDEMAN (1991). Soil erosion, soil degradation and climatic change. Dans : Land use changes in Europe. Processes of change, environmental transformations and future patterns. BROWER F.M., A.J. THOMAS et M.J. CHADWICK (Éditeurs), Kluwer Academic Publishers, Dordrecht, Pays-Bas, Chap. 12, pp. 275-292.

DICKINSON A. et P. BOLTON (1992). A programme of monitoring sediment transport in North Central Luzon, Philippines. Dans : Erosion and Sediment Transport Monitoring Programmes in River Basins. BOGEN J., D.E. WALLING et T.J. DAY (Éditeurs), IAHS Publication, No 210, Wallingford, Grande-Bretagne, pp. 483-493.

DUBREUIL P. et J. GUISCAFRE (1971). La planification du réseau hydrométrique minimal. Cahiers ORSTOM, Série hydrologie, 8, 3-38.
ELAHCENE O. et B. REMINI (2009). Corrélation entre la concentration en matière en suspension et le débit liquide dans le bassin versant d'oued Bellah (Algérie). Eur. J. Sci. Res., 26, 139-46.

ELAHCENE O., A.TERFOUS, B. REMINI, A. GHENAIM et J. POULET (2012). Étude de la dynamique sédimentaire dans le bassin versant de l'oued Bellah (Algérie). Hydrol. Sci. J., $119,1-17$.

ETCHANCHU D. et J.L. PROBST (1986). Érosion et transport de matières en suspension dans un bassin versant en région agricole. Méthode de mesure du ruissellement superficiel, de sa charge et des deux composantes du transport solide dans un cours d'eau. C. R. Acad. Sci. Paris, 302, Ser. II, 17, 1063-1067.

GHENIM A. (2001). Contribution à l'étude des écoulements liquides et des dégradations du bassin versant de la Tafna: cas d'oued Isser, oued Mouilah et la Haute Tafna. Thèse de maîtrise, Univ. Tlemcen, Algérie, 192 p.

GHENIM A. (2008). Étude des écoulements et des transports solides dans les régions semi-arides méditerranéennes. Thèse de doctorat, Univ. Abou-Bekr Belkaid, Algérie, 134 p.

HEIDEL S.G. (1956). The progressive lag of sediment concentration with flood waves. Eos, Trans. Amer. Geophys. Union, 37 (1), 56-66.

HEUSCH B. et A. MILLIES-LACROIX (1971). Une méthode pour estimer l'écoulement et l'érosion dans un bassin. Application au Maghreb. Mine et Géologie (Rabat), 33, 21-39.

IRVINE K.N. et J.J. DRAKE (1987). Process oriented estimation of sediment suspended concentration. Water Resour. Bull., 23, 1017-1025.

KATTAN Z., J.Y. GAC et J.L. PROBST (1987). Suspended sediment load and mechanical erosion in the Senegal basin, estimation of the runoff concentration and relative contributions of channel and slope erosion. J. Hydrol., 92, 59-76.

LAHLOU A. (1994). Envasement des barrages au Maroc. Collection "Sciences et techniques ", Édition Wallada, Casablanca, Maroc, 286 p. 
MEDJBER A. (2011). Influence de la variabilité climatique (précipitations) sur le taux de sédimentation dans certains barrages algériens. International Scientific Workshop: Relation man/environment and sediment transport: a spatial approach, 7 et 8 juin, Tipaza, Algeria.

MEGNOUNIF A. (2007). Étude du transport des sédiments en suspension dans les écoulements de surface. Thèse de doctorat, Univ. Abou-Bekr Belkaid, Algérie, 184 p.

MEGNOUNIF A., A. TERFOUS et S. OUILLON (2013). A graphical method to study suspended sediment dynamics during flood events in the Wadi Sebdou, NW Algeria (1973-2004). J. Hydrol., 497, 24-36.

MEGUENNI K. et B. REMINI (2008). Évaluation du débit solide dans le bassin versant de Harreza (Algerie). Larhyss J., 07, 7-19.

PEART M.D. et D.E. WALLING (1982). Particle size characteristics of fluvial suspended sediment. Dans : Recent developements in the explanation and prediction of erosion and sediment yield. WALLING D.E. (Éditeur), IAHS Publication, No 137, Wallingford, Grande-Bretagne, pp. 397-407.

PROBST J.L. et P. AMIOTTE SUCHET (1992). Fluvial suspended sediment transport an mechanical erosion in the Maghreb. Hydrol. Sci. J., 37 (6), 621-637.

REMENIERAS G. (1976). L'hydrologie de l'ingénieur. Collection : Direction des études et recherches d'Électricité de France (EDF), Eyrolles, Paris, France, 465 p.

SHABAN A. et M. KHAWLIE (1998). Geoenvironmental assessment of riparian zones under extreme climatic events: a case study of representative rivers in Lebanon. Mediterraneen Rivers and Riparian Zones-processes and Management Symposium, 21 septembre au 2 octobre, Saragosse, Espagne, 25 p.
SMITH H.G. et DRAGOVICH (2009). Interpreting sediment delivery processes using suspended sediment discharge hysteresis patterns from nested upland catchments, south eastern Australia. Hydrol. Process., 23 (17), 2415-2426.

SOGREAH (1967). Étude générale des aires d'irrigation et d'assainissement agricole en Algérie. Dossier, Ministère de l'agriculture et de la réforme agraire, Algérie, Mémoire de l'Institut de Géologie du Bassin d'Aquitaine, Bordeaux, France, $\mathrm{N}^{\circ} 22$.

STEPHEN T. (2000). Process, from and change in dry land rivers: a review of recent research. Earth-Sci. Rev., 51, 67107.

TERFOUS A., A. MEGNOUNIF et A. BOUANANI (2001). Étude du transport solide en suspension dans l'oued Mouillah. Rev. Sci. Eau, 14, 175-87.

TOUAIBIA B., A. AIDAOUI, D. GOMER et M. ACHITE (2001). Quantification et variabilité temporelles de l'écoulement solide en zone semi-aride de l'Algérie du Nord. Hydrol. Sci. J., 46 (1), 41-53.

WALLING D.E. (1984). The sediment yield of African rivers. Dans: Challenges in African hydrology and water resources. Walling D.E., S.S.D. Foster et P. Wurzel (Éditeurs), IAHS Publication, $\mathrm{N}^{\circ}$ 144, Wallingford, Grande-Bretagne, pp. 65-283.

WILLIAMS G.P. (1989). Sediment concentration versus water discharge during single hydrologic events in rivers. J. Hydrol., 111, 89-106.

WOOD P.A. (1977). Sediment transport in the Hope River, Jamaïca: a tropical drainage basin characterized by seasonal flow. Dans : Erosion and Solid Matter Transport in Inland Waters., IAHS Publication, No 122, Wallingford, GrandeBretagne, pp. 149-156. 